\title{
Is There a Difference in the Clinical Efficacy of Diosmin and Micronized Purified Flavonoid Fraction for the Treatment of Chronic Venous Disorders? Review of Available Evidence
}

\author{
Michèle Cazaubon' \\ Jean-Patrick Benigni ${ }^{2}$ \\ Marcio Steinbruch ${ }^{3}$ \\ Violaine Jabbour ${ }^{4}$ \\ Christelle Gouhier-Kodas ${ }^{4}$ \\ 'Private Vascular Specialist, Paris, France; \\ ${ }^{2}$ Pedagogical Support Office, Faculty of \\ Medicine Pitié-Salpétrière, Sorbonne \\ University, Paris, France; ${ }^{3}$ Hospital Albert \\ Einstein, São Paulo, Brazil; ${ }^{4}$ Laboratoire \\ Innotech International, Arcueil, France
}

\begin{abstract}
Flavonoids are oral venoactive drugs frequently prescribed to relieve the symptoms of chronic venous disorders (CVD). Among venoactive drugs, diosmin is a naturally occurring flavonoid glycoside that can be isolated from various plant sources; it can also be obtained after conversion of hesperidin extracted from citrus rinds. Micronized purified flavonoid fraction (MPFF) is a preparation that contains mainly diosmin and a small fraction of hesperidin. We performed a state-of-the-art literature review to collect and analyze wellconducted randomized clinical studies comparing diosmin - also called non-micronized or hemisynthetic diosmin - $600 \mathrm{mg}$ a day and MPFF, $1000 \mathrm{mg}$ a day. Three clinical studies met the criteria and were included for this literature review. These clinical studies showed a significant decrease of CVD symptom intensity (up to approximately 50\%) and global patient satisfaction after one-to-six-month treatment with diosmin or MPFF, without statistical differences between these two forms of diosmin. Both treatments were well tolerated with few mild adverse drug reactions reported. Overall, based on this literature review, there is no clinical benefit to increase the dose of diosmin beyond $600 \mathrm{mg}$ per day, to use the micronized form, or to add hesperidin, since clinical efficacy on venous symptomatology is achieved with $600 \mathrm{mg}$ per day of pure non-micronized diosmin. This challenges the status of diosmin - $600 \mathrm{mg}$ a day - in guidelines for the management of CVD, which is currently categorized $2 \mathrm{C}$ (weak recommendations for use and poor quality of evidence), while the most widely used and assessed preparation MPFF is rated $1 \mathrm{~B}$ (strong recommendation for use and moderate quality of evidence).
\end{abstract}

Keywords: chronic venous disorders, venoactive drugs, flavonoids, randomized clinical trials, literature review

\section{Introduction}

Chronic venous disorders (CVD) can be defined as morphological and functional abnormalities of the venous system that manifest either by symptoms and/or clinical signs. CVD is generally diagnosed on the basis of medical history, clinical examination and duplex color ultrasound. ${ }^{1}$ The main symptoms of CVD are feeling of swelling, heaviness and tightness in the legs, or pain in the lower limbs, and they increase in standing position and in summer. ${ }^{2}$ The symptoms are persistent and the disease is progressive, resulting in physical, social and psychological suffering with significant negative impact on quality of life. ${ }^{1,2}$
Correspondence: Christelle Gouhier-

Kodas

Email christelle.gouhier-

kodas@innothera.com 
The CEAP (Clinical Etiology Anatomy Pathophysiology) classification of CVD is widely used to describe CVD stages, based on clinical manifestations and other characteristics of the disease. ${ }^{3}$ The $\mathrm{C} 0-\mathrm{C} 3$ stages are not associated with visible trophic disorders: $\mathrm{C} 0$, no signs; $\mathrm{C} 1$, telangiectasias or reticular veins; $\mathrm{C} 2$, varicose; $\mathrm{C} 3$, oedema. Skin changes are visible at the next stages: $\mathrm{C} 4$, stasis dermatitis, pigmentation, lipodermatosclerosis, atrophie blanche or corona phlebectatica; $\mathrm{C} 5$, healed ulceration; C6, active ulceration. The descriptor "a" or " $\mathrm{s}$ " can be added after the $\mathrm{C}$ clinical class (asymptomatic or symptomatic) as at each CEAP stage, symptoms can be present or absent. ${ }^{4}$ Chronic venous insufficiency refers to the most advanced stages of the disease. Venous ulcers, the ultimate stage of CVD, are present in $1-2 \%$ of the Northwestern population and are a heavy burden, both for patients and public health systems. ${ }^{1}$

The precise etiology and pathogenesis of CVD remain incompletely understood, although a variety of possibly inter-related mechanisms have been proposed to be responsible for signs and symptoms, such as venous valve incompetence, structural alterations and changes in cellular components of the venous wall, inflammation of the venous wall and an increase in venous pressure. ${ }^{5,6}$ The pooling of blood in the lower extremity (prolonged standing position in normal subjects or venous incompetence in ill subjects) leads to an increase in hydrostatic pressure and in the blood flow in capillaries. The resulting venous hypertension prompts leucocyte adhesion to capillary endothelium and initializes an inflammatory reaction. The expression and activity of hypoxia-inducible factors, cytokines, reactive oxygen species or metalloproteinases increase. The extracellular matrix, which is essential for the endothelial cells' nutrition and homeostasis, is altered. These mechanisms result in progressive dilation of the venous wall, valve incompetence and venous reflux, which may play a role in the onset of symptoms. Venous pathology could then progress through a vicious cycle of inflammation and leukocyte recruitment leading to further deteriorations. $^{5,7-9}$

The choice of treatment for CVD depends on the CEAP stages and different treatments are used to meet several objectives. ${ }^{10}$ Graduated compression stockings help fighting against venous stasis, reduce symptoms and might prevent disease progression. Oral venoactive drugs have various pharmacological actions, notably targeted at the venous wall, and are used to relieve symptoms. Creams or lotions can also be helpful to relieve symptoms.
In the most advanced stages, sclerotherapy, endovenous procedures and surgery can be done to remove pathologic veins. Finally, compressive bandages improve circulation in deep veins and microvascular structures by reducing swelling and improving skin nutrition. ${ }^{1}$

Oral treatments are frequently prescribed to patients suffering from symptomatic CVD. Venoactive drugs are a heterogeneous pharmacological class that comprises both synthetic and compounds extracted from plants. Among venoactive drugs, flavonoids are a class of polyphenolic compounds found in plants, and many studies have suggested that they were effective for the treatment of symptomatic CVD. Although the action of flavonoids is not fully understood, they were shown to increase the venous tone by modulating noradrenergic signaling. Moreover, in vitro and in vivo investigations suggested they could target primarily the venous wall through anti-inflammatory and antioxidant properties, which may be their key mechanisms of action. ${ }^{11,12}$

Diosmin (diosmetin 7-O-rutinoside) is a naturally occurring flavonoid glycoside that can be isolated from various plant sources or derived from hesperidin, another flavonoid which is chemically and structurally very similar. ${ }^{12}$ Various specialties containing diosmin are available, including micronized purified flavonoid fraction (MPFF) which contains $90 \%$ diosmin and $10 \%$ hesperidin. Micronization is a mechanical process that reduces the average diameter of solid particles to improve intestinal absorption. ${ }^{13}$

Based on systematic reviews or meta-analyses of clinical studies looking at the effects of individual venoactive drugs on symptoms of CVD, MPFF was shown to be effective on improving leg symptoms, oedema, and quality of life in patients with CVD. ${ }^{14-16}$ Therefore, successive international guidelines for CVD management presented MPFF as the flavonoid preparation that achieved the highest level of recommendation, and this drug is currently considered as the reference treatment among flavonoid-based venoactive drugs. ${ }^{11,15}$ Nevertheless, the meta-analyses on which these recommendations were based on did not include the clinical studies comparing diosmin, also called non-micronized or hemisynthetic diosmin, $600 \mathrm{mg}$ a day and MPFF $1000 \mathrm{mg}$ a day. As MPFF treatment is strongly recommended, it is worth considering other therapeutics that have been compared in terms of efficacy to this reference product. The objective of this literature review was therefore to collect and analyze randomized clinical studies with appropriate methodology comparing these two diosmin preparations, and then to assess if these studies provide sufficient robust 
evidence to consider non-micronized diosmin - $600 \mathrm{mg}$ a day - in international recommendations.

\section{Methods}

\section{Criteria for Considering Studies for This Review}

Only randomized clinical studies comparing the efficacy of approved doses of non-micronized diosmin or MPFF in their relevant therapeutic indication (venous symptom improvement) were selected: $600 \mathrm{mg}$ per day in one intake for non-micronized diosmin and $1000 \mathrm{mg}$ per day in one or two intakes for MPFF.

\section{Search Method for Identification of Studies}

Bibliographic databases (PubMed, Web of Science, Google Scholar) were searched from February 11th, 2021 to July 8th, 2021, using different keywords ("chronic venous disease", "chronic venous insufficiency", "flavonoids", "diosmin", "micronized purified flavonoid fraction", "randomized clinical trial", combined with logical AND and OR connectors). Only articles reporting clinical trials were selected; there were no limitations on language or publication date; studies from all countries were eligible for inclusion. Recent reviews, meta-analyses and personal bibliographies of experts were also consulted to complete the literature review.

Study selection was conducted in two stages: an initial screening of titles and/or abstracts to identify potentially relevant papers, followed by screening of the full-text papers identified during the initial stage.

\section{Results}

\section{Search Results}

After removing duplicate citations from the retrieved records, 285 articles were identified for further screening. A total of five records describing randomized clinical trials comparing the efficacy of non-micronized diosmin and MPFF in their relevant therapeutic indication (venous symptom improvement) were selected, and full appraisal of these publications was carried out (Figure 1).

\section{Selected Studies}

Among the five publications considered eligible for analysis of the full text, only three blinded, randomized trials comparing non-micronized diosmin and MPFF were selected for inclusion in the review, namely the studies of Henriet et al, ${ }^{17}$ Maruszynski et $\mathrm{al}^{18}$ and Steinbruch et $\mathrm{al}^{19}$ (Table 1). Two studies met the inclusion criteria for the literature review but were excluded after analysis of the full article. The Delecluse et al study (1991) - comparing non-micronized diosmin $300 \mathrm{mg}$ twice a day and MPFF $500 \mathrm{mg}$ twice a day in a singleblind scheme - concluded to a comparable efficacy of both treatments but was rejected because no statistical analysis was conducted to compare treatment groups. ${ }^{20}$ The Cospite et al study (1989) - that concluded to therapeutic advantage of MPFF - was also discarded due to important methodological concerns: MPFF was compared to non-micronized diosmin given at a lower dose than that generally recommended for approved nonmicronized diosmin products for CVD treatment, no details on blinding and on galenics of the diosmin

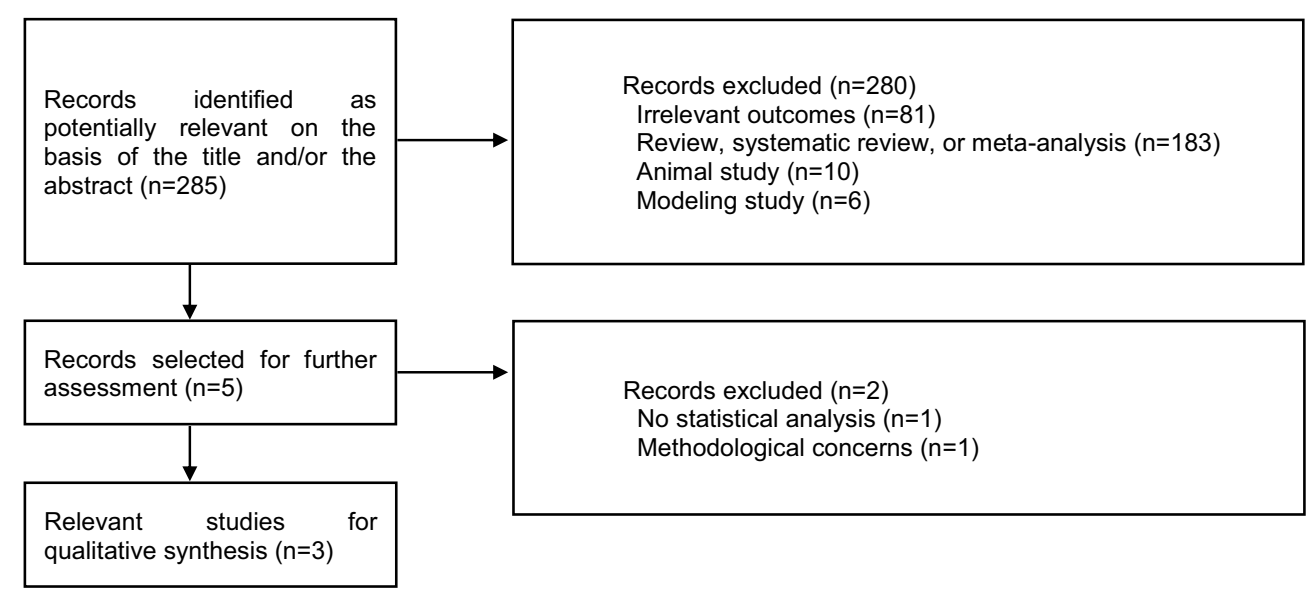

Figure I Flow Diagram of the Literature Review 


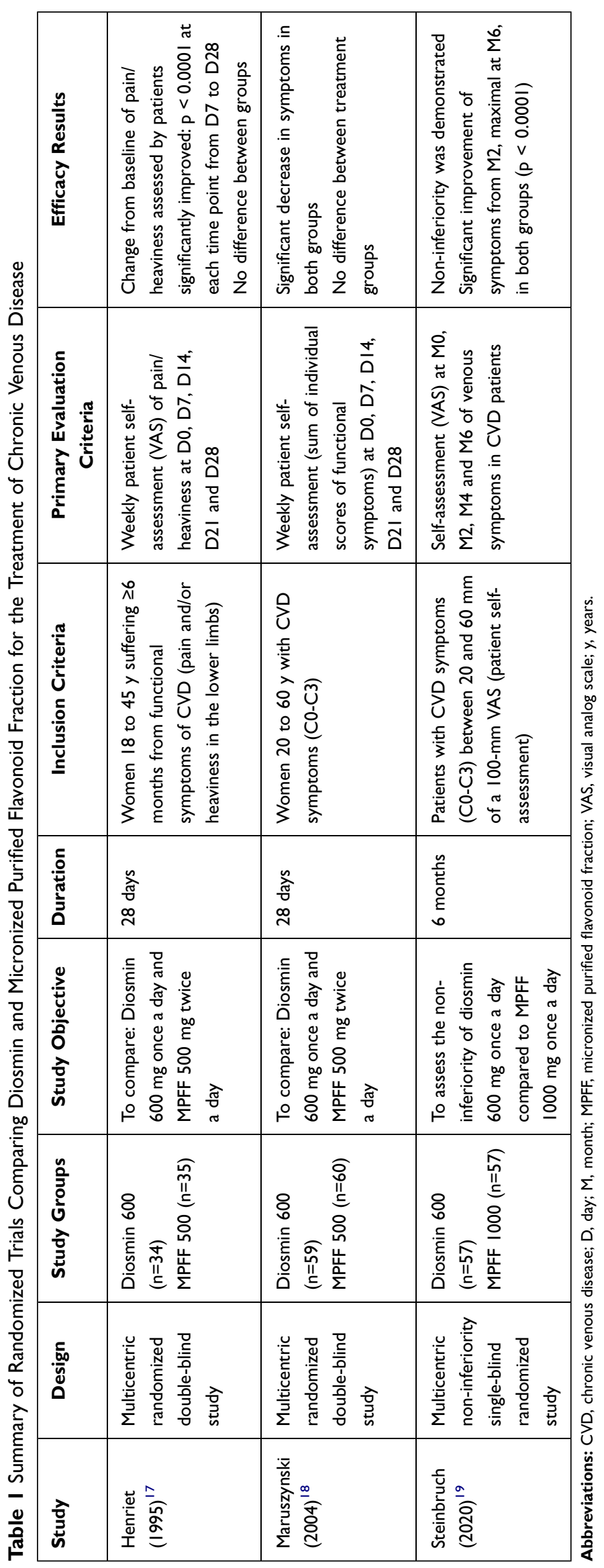


comparator were provided. ${ }^{21}$ This latter study has never been included in a systematic review or meta-analysis. ${ }^{22}$

In 1995, Henriet et al conducted a Phase III clinical study to compare the efficacy of non-micronized dios$\min 600 \mathrm{mg}$ (Diovenor ${ }^{\circledR} 600 \mathrm{mg}$ ) once a day vs MPFF $500 \mathrm{mg}$ (Daflon ${ }^{\circledR} 500 \mathrm{mg}$ ) twice a day in CVD. ${ }^{17}$ It was a prospective, comparative, randomized, double-blind, double-dummy study performed in nine French centers in women aged 18 to 45 years old suffering from pain and/or heaviness in the two lower limbs for at least 6 months. Women showed no hemodynamic signs, no oedema or varicose veins. Patients were randomized to receive non-micronized diosmin $600 \mathrm{mg}$ once a day or MPFF $500 \mathrm{mg}$ twice a day (plus placebos). The primary endpoints were weekly patient self-assessment (visual analog scale; VAS) at D0, D7, D14, D21 and D28 and a clinical functional score assessed by the physician (sum of limb symptom scores: pain/heaviness, feeling of swelling, paraesthesia, each from 0 to 3). Secondary endpoints were overall effectiveness as judged by physicians' and patients' opinion. Sixty-nine women (mean age, 30 years) were randomized: 34 received nonmicronized diosmin and 35 MPFF.

Later, Maruszynski et al compared the clinical efficacy of hemisynthetic diosmin with MPFF in women with CVD symptoms in a multicenter, randomized, double-blind, double-dummy study. ${ }^{18}$ Women aged $20-60$ years with CEAP class C0s-C3 CVD were included in three centers in Poland. The primary endpoint was the self-assessment of therapy effectiveness using a questionnaire based on a visual scale of lower limb symptom intensity. The selfassessment questionnaire consisted of six questions concerning: swelling, feeling of heaviness, leg tiredness, heat, night muscle cramps and pain. Symptom intensity, assessed on a scale from 0 to 5 points for each symptom, was recorded at D0, D7, D14, D21 and D28. Women were included in the study if they achieved a total score $\geq 6$ points to the self-assessment questionnaire at the initial evaluation. The other evaluation criteria were the overall assessment according to the patient (satisfaction) and according to the physician (adequacy of the treatment to the clinical situation) at the check-up visit. Fifty-nine patients received hemisynthetic diosmin $600 \mathrm{mg}$ once a day $\left(\right.$ Otrex $\left.^{\circledR} 600\right)$ plus one placebo and 60 received MPFF $500 \mathrm{mg}$ twice a day $\left(\right.$ Detralex $\left.^{\circledR}\right)$. The use of compression devices was not allowed during the study.

Finally, a recent study conducted by Steinbruch et al assessed the clinical non-inferiority of non-micronized diosmin (Flebodia ${ }^{\circledR} 600 \mathrm{mg}$ ) compared to MPFF (Daflon ${ }^{\circledR}$ $1000 \mathrm{mg}$ ) over a 6-month treatment period. ${ }^{19}$ In this prospective, randomized, single-blind study, adult patients with symptomatic CVD of the lower limbs (CEAP classification from C0s-C3 stages; $20-60 \mathrm{~mm}$ on 100-mm VAS) were included. The primary endpoint was the self-assessment of the leg symptom intensity on a VAS from baseline to 6 months. Secondary endpoints were the overall satisfaction of the patients and physicians, the difficulty to swallow the pills assessed using a VAS, and the treatments' safety. A total of 114 patients (mean age, 44.4 years; women: 90.4\%) were included in six Brazilian university centers and randomized in the non-micronized diosmin group $(\mathrm{n}=57$, one $600 \mathrm{mg}$ tablet once a day) and the MPFF group ( $\mathrm{n}=57$, one $1000 \mathrm{mg}$ tablet once a day). All were included in the per-protocol analysis.

\section{Efficacy of Diosmin Compared to MPFF in the Treatment of CVD}

These three blinded randomized trials comparatively assessed the efficacy of non-micronized diosmin $600 \mathrm{mg}$ a day and MPFF $1000 \mathrm{mg}$ a day on specific and/or global symptoms of CVD as detailed below. Since the medicines containing one of these two forms of diosmin are generally indicated for venous symptom improvement, clinical signs were not considered in this review.

\section{Global Symptomatology}

A significant reduction in the global intensity of CVD symptoms was shown in the three studies after one to six months of treatment with non-micronized diosmin $600 \mathrm{mg}$ and MPFF $1000 \mathrm{mg}$, with no statistical differences between the two treatment groups. In the study conducted by Henriet et al, a global index of functional symptoms was determined by the physicians using the sum of the scores for three different symptoms - heavy legs and/or pain, sensation of lower limb oedema and paraesthesia each symptom was scored from 0 to 3 (the highest score indicating a strong impact on the patients' quality of life). ${ }^{17}$ The two treatment groups were comparable at baseline with a mean score of $4.18 \pm 0.51$ for the nonmicronized diosmin $600 \mathrm{mg}$ group and $4.91 \pm 0.62$ for the MPFF $1000 \mathrm{mg}$ group. After one month of treatment, this index of functional symptoms was globally improved versus baseline in the two groups, with a reduction of 2.53 \pm 0.63 and $3.29 \pm 0.68$, respectively $(\mathrm{p}<0.0001)$.

Similarly, a global score of symptoms was used to determine the efficacy of the non-micronized diosmin 
$600 \mathrm{mg}$ compared to MPFF $1000 \mathrm{mg}$ in the study of Maruszynski et al. ${ }^{18}$ This global score was calculated using a patient self-assessment questionnaire based on six symptoms of CVD (ie, swelling of the calf, sensation of leg heaviness, sensation of leg tiredness, heat sensation in legs, night muscle cramps and pain), each scored on a six-point scale (0: No symptom - 5: Extremely intense symptom). Baseline scores were comparable between the two groups, and a significant decrease in this global score was observed at each following visit, ie, at D7, D14, D21 and D28, with no statistical differences between the two groups. The physician's assessment of symptoms using the same scale also showed that there was no difference in terms of efficacy between treatments.

The comparable general efficacy of non-micronized diosmin $600 \mathrm{mg}$ and MPFF $1000 \mathrm{mg}$ was recently confirmed by Steinbruch et al using another rating scale. ${ }^{19}$ A $100 \mathrm{~mm}$ VAS was used by patients to describe the intensity of their lower limb's symptoms at the first visit and after two, four and six months of treatment. At the first visit, the mean (SD) intensity of venous symptoms was rated 47.1 (8.2) $\mathrm{mm}$ in the non-micronized diosmin group and $50.3(9.5) \mathrm{mm}$ in the MPFF group. Symptoms improved significantly at the end of the treatment course, with adjusted mean VAS changes of $-24.9 \mathrm{~mm}(\mathrm{p}<0.0001)$ in the non-micronized diosmin group and $-22.8 \mathrm{~mm}(\mathrm{p}<0.0001)$ in the MPFF group corresponding to an approximately $50 \%$ reduction in basal symptom intensity. More specifically, according to the results of this study, there was a $95 \%$ chance that the symptomatologic improvement from baseline with nonmicronized diosmin was, at worst, $1 \mathrm{~mm}$ lower than the improvement with MPFF on the $100 \mathrm{~mm}$ VAS, therefore, demonstrating the non-inferiority of the non-micronized diosmin treatment.

\section{Pain}

In two of the analysed studies, the efficacy of treatments to reduce pain associated with CVD was evaluated independently of other symptoms. In the study published by Henriet et al, the mean baseline pain intensity, evaluated by patients using a $100 \mathrm{~mm}$ VAS, was estimated to be $66.82 \pm 6.66 \mathrm{~mm}$ and $63.42 \pm 7.44 \mathrm{~mm}$ in the nonmicronized diosmin and the MPFF groups, respectively. ${ }^{17}$ A significant and equivalent reduction was observed in both groups, reaching $-40.09 \pm 7.08 \mathrm{~mm}$ and $-34.90 \pm$ $10.81 \mathrm{~mm}(\mathrm{p}<0.0001)$, respectively.

Consistent results were obtained in the study conducted by Maruszynski et al, in which, at the first visit, pain was scored by patients, on a 0 to 5 point scale, to be $1.6 \pm 1.2$ in the nonmicronized diosmin group and $1.6 \pm 1.3$ in the MPFF group. ${ }^{18}$ After 28 days of treatment, scores of pain in the legs were reduced similarly in both groups, by approximately 1 point, to reach very low pain intensity levels.

\section{Feeling of Leg Swelling}

An evaluation of the patients' sensation of swelling and of the leg swelling by the physician, using a $0-5$ point scale, was performed before and after 1 month of treatment with non-micronized diosmin $600 \mathrm{mg}$ or MPFF $100 \mathrm{mg}$ in the study conducted by Maruszynski et al. ${ }^{18}$ Patients reported a significant and comparable decrease in the sensation of swelling of around 1.2 point in both groups (baseline scores: $3.2 \pm 1.1$ and $2.8 \pm 1.1$ points in the non-micronized diosmin and MPFF groups respectively). The investigators also observed a decrease in leg swelling intensity after 1 month of treatment, with no statistical differences between groups.

\section{Feeling of Leg Heaviness and Tiredness}

The sensations of heaviness and tiredness in the legs have also been evaluated separately from other CVD symptoms in the study of Maruszynski et al. ${ }^{18}$ Before treatment initiation, patients estimated, on a scale from 0 to 5 points, the scores corresponding to their feeling of heaviness to be $3.2 \pm 1.1$ and $2.8 \pm 1.1$ and that corresponding to their sensation of tiredness to be $3.0 \pm 1.1$ and $3.1 \pm 1.0$ for the non-micronized diosmin $600 \mathrm{mg}$ and the MPFF $1000 \mathrm{mg}$, respectively. These scores decreased by about 1.6 points after 28 days of treatment in the two groups.

\section{Night Cramps}

Patients suffering from CVD can be subject to muscle cramps. $^{23}$ Maruszynski and his collaborators evaluated the impact of the two treatments on the occurrence of cramps in their patients, using the scale previously described (from 0 to 5 points). ${ }^{18}$ Results suggested that non-micronized diosmin $600 \mathrm{mg}$ tends to reduce more efficiently night cramps than MPFF $1000 \mathrm{mg}(\mathrm{p}=0.022$ on $\mathrm{D} 14, \mathrm{p}=0.096$ on $\mathrm{D} 21, \mathrm{p}=0.074$ on $\mathrm{D} 28$ ).

\section{Safety of Diosmin Compared to MPFF in the Treatment of CVD}

In all the studies analysed in this review, treatment-emergent adverse events, as well as study withdrawals due to adverse events, were monitored. The most common adverse reactions reported were gastrointestinal disorders (Table 2).

In the Henriet et al study, three out of 34 patients from the non-micronized diosmin group reported 
Table 2 Summary of Adverse Reactions Reported During the Randomized Trials

\begin{tabular}{|c|c|c|}
\hline Study & Non-Micronized Diosmin 600 mg & MPFF 1000 mg \\
\hline $\begin{array}{l}\text { Henriet } \\
(1995)^{17}\end{array}$ & Vomiting and diarrhoea $(n=I)$, gastralgia $(n=I)$, headache $(n=I)(n=3 / 34)$ & $\begin{array}{l}\text { Gastralgia }(n=3) \text {, constipation and abdominal } \\
\text { meteorism }(n=1) \text {, nausea }(n=1)(n=5 / 35)\end{array}$ \\
\hline $\begin{array}{l}\text { Maruszynski } \\
(2004)^{18}\end{array}$ & Calf, hand and feet oedema, body rash $(n=N 1 / 59)$ & Calf oedema, body rash, mouth dryness $(n=N I / 60)$ \\
\hline $\begin{array}{l}\text { Steinbruch } \\
(2020)^{19}\end{array}$ & $\begin{array}{l}\text { Nausea }(13.3 \%) \text {, dyspepsia }(16.7 \%) \text {, diarrhoea }(8.3 \%) \text {, headache }(6.7 \%) \text {, } \\
\text { vertigo }(6.7 \%)(n=21 / 57)\end{array}$ & $\begin{array}{l}\text { Nausea }(20 \%) \text {, dyspepsia }(6.7 \%) \text {, diarrhoea }(5.0 \%) \text {, } \\
\text { headache }(6.7 \%) \text {, vertigo }(3.3 \%)(n=16 / 57)\end{array}$ \\
\hline
\end{tabular}

Abbreviations $\mathrm{n}$, Number of patients experiencing adverse reaction; NI, Not indicated; MPFF, micronized purified flavonoid fraction.

gastralgia or headache, one of them dropped out of the trial after experiencing vomiting and diarrhoea. ${ }^{17}$ In the MPFF group, two out of 35 patients withdrew the trial due to gastralgia, and three others reported adverse drug reactions without discontinuing the treatment (constipation and abdominal meteorism for one patient, gastralgia or nausea for the two others). In the study conducted by Maruszynski et al, adverse reactions with nonmicronized diosmin $600 \mathrm{mg}$ were of two types: Calf, hands and feet oedema and body rash. In the MPFF $1000 \mathrm{mg}$ group, three reaction types have been reported: Calf oedema, body rash and mouth dryness. ${ }^{18}$ Three patients stopped their treatment after the occurrence of mild adverse events, but the authors did not specify to which study group they belonged to. No serious adverse drug reaction was reported in the Steinbruch et al study. ${ }^{19}$ The percentage of patients with adverse reactions was $35.0 \%$ in the non-micronized diosmin $600 \mathrm{mg}$ group and $26.7 \%$ in the MPFF $1000 \mathrm{mg}$ group. Dyspepsia, nausea, diarrhoea and headache were the most frequently reported reactions in the two groups. Two patients discontinued their treatment with nonmicronized diosmin because of palpitations, abdominal pain, diarrhoea and/or dyspepsia. In the MPFF group, one patient stopped his treatment due to vertigo and weight decrease.

\section{Discussion}

Venoactive drugs in CVD have been assessed in clinical studies that were generally small in size. Therefore, conclusions on the efficacy of these treatments are mainly based on meta-analyses and expert consensus statements. A Cochrane review and meta-analysis of phlebotonics for venous insufficiency, published in 2020, analyzed 56 randomized, double-blind, placebo-controlled trials involving 7690 participants. ${ }^{24}$ Ten trials with MPFF out of forty using flavonoids were included. ${ }^{24}$ The meta-analysis suggests that oral venoactive drugs considered as a whole reduce lower leg oedema (risk ratio (RR) $0.70,95 \%$ confidence interval (CI) 0.63 to 0.78 ) and ankle circumference (mean difference $-4.27 \mathrm{~mm}, 95 \% \mathrm{CI}-5.61$ to $-2.93 \mathrm{~mm}$ ) compared with placebo. Little or no effect was evidenced for ulcer healing (RR $0.94,95 \%$ CI 0.79 to 1.13 ).

In the 2005 International Consensus Statement, 83 randomized studies and meta-analyses were considered, and experts evaluated the level of evidence-based medicine using three levels of recommendations, A, B and $\mathrm{C}$ (from large randomized clinical trials to nonrandomized trials). Grade A was attributed to MPFF, calcium dobesilate and hydroxyethyl-rutosides, Grade B to Ruscus extracts and Grade $\mathrm{C}$ to hemisynthetic diosmin. ${ }^{15}$ The 2008 guidelines for the management of CVD updated the conclusions of published Cochrane reviews and 2005 International Consensus Statement (no significant changes). ${ }^{25}$

Perrin and Ramelet published in 2011 a literature review to evaluate venoactive drugs using the GRADE system. ${ }^{26}$ In this system, recommendations are graded from 1 to 3 and level of evidence from A to C. Grade $1 \mathrm{~B}$ was attributed to MPFF and rutosides and Grade $2 \mathrm{C}$ to Ruscus extracts. When the guidelines for the use of venous active drugs were updated in 2014 , it was proposed to quantify the level of recommendations with the GRADE system. ${ }^{27}$ MPFF received 1B grade (strong recommendation for use and moderate quality of evidence) and nonmicronized diosmin or synthetic diosmin received $2 \mathrm{C}$ grade (low recommendations and poor quality of evidence). Rutosides and Ruscus extracts received 2B grade.

In the recent 2018 Guidelines According to Scientific Evidence, published by Nicolaides and coworkers, it was proposed to update the 2014 guidelines by individualizing both symptoms and venous active drugs with ancient and 
recent data. ${ }^{11}$ However, only five venoactive specialties were considered: MPFF, Ruscus extract plus hesperidin methyl chalcone and ascorbic acid, hydroxyethyl-rutosides, horse chestnut seed extract and calcium dobesilate. There was no analysis of clinical trials with nonmicronized or hemisynthetic diosmin.

Head-to-head comparisons are rare in clinical trials evaluating venoactive drugs. In addition, meta-analyses preferentially select placebo-controlled studies. ${ }^{22,24,28}$ However, since both MPFF and non-micronized diosmin are flavonoids and MPFF is mostly composed of diosmin, these compounds are expected to share comparable pharmacological properties. Both are known to intervene in several of the pathophysiological mechanisms of CVD. Diosmin directly acts on venous tone by enhancing sympathetic-mediated venous contractibility and increasing calcium sensitivity and contractility. $^{29,30}$ Furthermore, MPFF and non-micronized diosmin have demonstrated a broad anti-inflammatory activity through the inhibition of leukocyte adhesion to the vascular endothelium and the reduction of pro-inflammatory factor expression (eg, TNF- $\alpha$, IL-6). ${ }^{31,32}$

This literature review identified three well-conducted multicenter randomized clinical trials with appropriate methodology comparing MPFF and non-micronized diosmin and providing consistent results. Taken all together, they indicate that clinical symptoms of CVD are improved with both treatments and that there are no significant differences between these two diosmin preparations. The recent study of Steinbruch et $\mathrm{al}^{19}$ was a 6-month non-inferiority trial, and its methodology was therefore very demanding (low rate of patients lost to follow-up, low rate of protocol deviations, consistent intent-to-treat and per-protocol analyses). Despite an intermediate number of patients, the results provide a persuasive demonstration of the non-inferiority of nonmicronized diosmin $600 \mathrm{mg}$ compared with MPFF $1000 \mathrm{mg}$ (diosmin $900 \mathrm{mg}$ plus hesperidin $100 \mathrm{mg}$ ) after a 6-month treatment period. Furthermore, overall satisfaction of patients and physicians was good and comparable in both groups. ${ }^{19}$ The studies of Henriet et al and Maruszynski et al have also individually proven that treatment with non-micronized diosmin $600 \mathrm{mg}$ or MPFF $1000 \mathrm{mg}$ were equally efficient to reduce the feeling of heaviness and pain in the lower limbs after one month of treatment. ${ }^{17,18}$ Similar consistent beneficial effects of diosmin and MPFF were found across different clinical outcomes measured either objectively (oedema) or subjectively (pain, sensation of heaviness or swelling, etc.) using various assessment tools, in placebo-controlled or non-comparative randomized studies. Statistically significant reduction in pain was observed gradually over the course of treatment with $\mathrm{MPFF}^{33,34}$ and diosmin ${ }^{35,36}$ versus baseline intensity level.

The three studies considered in this review agreed on the equally good safety profile of both diosmin preparations, without serious adverse events. The higher frequency of adverse reactions in the study conducted by Steinbruch et al, compared to that observed in the two other studies presented in this review, or in other clinical trials conducted with one of the two drugs could be explained by the longer treatment duration ( 6 months). The adverse events reported were mainly digestive disorders which are expected adverse reactions associated with diosmin preparations. ${ }^{24}$

\section{Conclusion}

Based on this literature review, MPFF (1000 mg once a day or $500 \mathrm{mg}$ twice a day) and non-micronized diosmin (600 mg once a day) appear to have comparable clinical effects on CVD symptoms, after a short treatment duration (1 month), and also after a long-term treatment (6 months). These results are not surprising since MPFF is made of $90 \%$ diosmin. They suggest that increasing the diosmin daily dose, enhancing its bioavailability with micronization, or adding another flavonoid fraction, hesperidin, do not substantially improve the relief of symptoms. Thus, it supports that the daily dose of $600 \mathrm{mg}$ diosmin is an equally valuable therapeutic option in this indication. Because clinical studies have been performed mostly with diosmin preparations in the form of MPFF, the recommendation for this flavonoid specialty is rated $1 \mathrm{~B}$ (strong recommendation for use and moderate quality of evidence), while it is rated $2 \mathrm{C}$ for hemisynthetic diosmin (weak recommendation for use and poor quality of evidence). Such a difference in grading between the two formulations may not be justified and the results of this literature review suggest that the place of non-micronized diosmin might be reevaluated in the future updates of guidelines for the management of CVD. Confirmation of the findings of this review by further randomized comparative studies might help support this reassessment.

\section{Disclosure}

Michèle Cazaubon agreed to review this article in exchange to a compensation provided by Laboratoire Innotech International. Jean-Patrick Benigni is currently under 
contract with Laboratoire Innotech International as a consultant expert providing advices on compression therapy and chronic venous disorders. Violaine Jabbour and Christelle Gouhier-Kodas are employed by Laboratoire Innotech International. Marcio Steinbruch declares that he has no conflict of interest regarding this publication. The authors report no other conflicts of interest in this work.

\section{References}

1. Shami SK, Sarin S, Scurr JH. Chronic venous insufficiency disease. Int J Angiol. 1997;6:30-48. doi:10.1007/BF01616230

2. Eberhardt RT, Raffetto JD. Chronic venous insufficiency. Circulation. 2014;130:333-346. doi:10.1161/CIRCULATIONAHA.113.006898

3. Porter JM, Moneta GL. Reporting standards in venous disease: an update. International consensus committee on chronic venous disease. $J$ Vasc Surg. 1995;21:635-645. doi:10.1016/S07415214(95)70195-8

4. Lurie F, Passman M, Meisner M, et al. The 2020 update of the CEAP classification system and reporting standards. J Vasc Surg. 2020;8:342-352.

5. Labropoulos N. How does chronic venous disease progress from the first symptoms to the advanced stages? A review. Adv Ther. 2019;36:13-19. doi:10.1007/s12325-019-0885-3

6. Santler B, Goerge T. Chronic venous insufficiency - a review of pathophysiology, diagnosis, and treatment. J Dtsch Dermatol Ges. 2017;15:538-556.

7. Raffetto JD, Khalil RA. Mechanisms of lower extremity vein dysfunction in chronic venous disease and implications in management of varicose veins. Vessel Plus. 2021;5:36.

8. Feldo M, Woźniak M, Wójciak-Kosior M, et al. Influence of diosmin treatment on the level of oxidative stress markers in patients with chronic venous insufficiency. Oxid Med Cell Longev. 2018;2018:2561705. doi:10.1155/2018/2561705

9. Serra R, Andreucci M, De Caridi G, et al. Functional chronic venous disease: a systematic review. Phlebology. 2017;32:588-592. doi: $10.1177 / 0268355516686451$

10. Mansilha A, Sousa J. Pathophysiological mechanisms of chronic venous disease and implications for venoactive drug therapy Int J Mol Sci. 2018;19:1669. doi:10.3390/ijms19061669

11. Nicolaides A, Kakkos S, Baekgaard N, et al. Management of chronic venous disorders of the lower limbs. Guidelines according to scientific evidence. part I. Int Angiol. 2018;37:181-254. doi:10.23736/ S0392-9590.18.03999-8

12. Rabe E, Guex -J-J, Morrison N, et al. Treatment of chronic venous disease with flavonoids: recommendations for treatment and further studies. Phlebology. 2013;28:308-319. doi:10.1177/ 0268355512471929

13. Garner RC, Garner JV, Gregory S, et al. Comparison of the absorption of micronized (Daflon $500 \mathrm{mg}$ ) and nonmicronized 14C-diosmin tablets after oral administration to healthy volunteers by accelerator mass spectrometry and liquid scintillation counting. J Pharm Sci. 2002;91:32-40. doi:10.1002/jps.1168

14. Kakkos SK, Nicolaides AN. Efficacy of micronized purified flavonoid fraction (Daflon ${ }^{\circledR}$ ) on improving individual symptoms, signs and quality of life in patients with chronic venous disease: a systematic review and meta-analysis of randomized double-blind placebocontrolled trials. Int Angiol. 2018;37:143-154. doi:10.23736/S03929590.18.03975-5

15. Ramelet AA, Boisseau MR, Allegra C, et al. Veno-active drugs in the management of chronic venous disease. An international consensus statement: current medical position, prospective views and final resolution. Clin Hemorheol Microcirc. 2005;33:309-319.
16. Allaert FA. Meta-analysis of the impact of the principal venoactive drugs agents on malleolar venous edema. Int Angiol. 2012;31:310-315.

17. Henriet J. Insuffisance veineuse fonctionnelle: essai clinique comparatif d'une seule prise par jour de Diovenor $600 \mathrm{mg}(600 \mathrm{mg}$ de diosmine d'hémisynthèse) versus 2 prises par jour d'un mélange de $500 \mathrm{mg}$ de flavonoïdes (900 $\mathrm{mg}$ de diosmine). [Functional venous insufficiency: comparative clinical trial of a single daily dose of DIOVENOR $600 \mathrm{mg}$ (600 $\mathrm{mg}$ of hemisynthetic diosmin) versus two daily doses of a combination of $500 \mathrm{mg}$ of flavonoids (900 $\mathrm{mg}$ of diosmin)]. Phlébologie (Annales Vasculaires). 1995;48:285-290.

18. Maruszynski A. A double blind, randomized study evaluating the influence of semisynthetic diosmin, and purified, micronized flavonoid fraction (diosmin and hesperidin), on symptoms of chronic venous insufficiency of lower limb - a four week observation. Przeglad Flebologiczny. 2004;13:89-95.

19. Steinbruch M, Nunes C, Gama R, et al. Is nonmicronized diosmin $600 \mathrm{mg}$ as effective as micronized diosmin $900 \mathrm{mg}$ plus hesperidin $100 \mathrm{mg}$ on chronic venous disease symptoms? Results of a noninferiority study. Int J Vasc Med. 2020;2020:4237204.

20. Delecluse M, Ducros JJ, Egal G, et al. Essai clinique pragmatique de Diovenor $300 \mathrm{mg}$ versus mélange de flavonoïdes à $90 \%$ de diosmine dans le traitement des manifestations d'insuffisance veineuse chronique chez la femme active jeune. [Pragmatic clinical trial of Diovenor $300 \mathrm{mg}$ versus a mixture of flavonoids with $90 \%$ diosmin in the treatment of symptoms of chronic venous insufficiency in young active women]. Artères et Veines. 1991;10:498-503.

21. Cospite M, Dominici A. Double blind study of the pharmacodynamic and clinical activities of $5682 \mathrm{SE}$ in venous insufficiency. Advantages of the new micronized form. Int Angiol. 1989;8:61-65.

22. Martinez-Zapata MJ, Vernooij RWM, Uriona Tuma SM, et al. Phlebotonics for venous insufficiency. Cochrane Database Syst Rev. 2016;4:CD003229.

23. Eklöf B, Rutherford RB, Bergan JJ, et al. Revision of the CEAP classification for chronic venous disorders: consensus statement. J Vasc Surg. 2004;40:1248-1252. doi:10.1016/j.jvs.2004.09.027

24. Martinez-Zapata MJ, Vernooij RWM, Simancas-Racines D, et al. Phlebotonics for venous insufficiency. Cochrane Database Syst Rev. 2020;3. doi:10.1002/14651858.CD003229.pub4

25. Nicolaides AN, Allegra C, Bergan J, et al. Management of chronic venous disorders of the lower limbs: guidelines according to scientific evidence. Int Angiol. 2008;27:1-59.

26. Perrin M, Ramelet AA. Pharmacological treatment of primary chronic venous disease: rationale, results and unanswered questions. Eur J Vasc Endovasc Surg. 2011;41:117-125. doi:10.1016/j.ejvs.2010.09.025

27. Nicolaides A, Kakkos S, Eklof B, et al. Management of chronic venous disorders of the lower limbs - guidelines according to scientific evidence. Int Angiol. 2014;33:87-208.

28. Martinez-Zapata MJ, Bonfill X, Moreno RM, Vargas E, Capellà D. Phlebotonics for venous insufficiency. Cochrane Database Syst Rev. 2005;3:CD003229. doi:10.1002/14651858.CD003229.pub2

29. Boudet C, Peyrin L. Comparative effect of tropolone and diosmin on venous COMT and sympathetic activity in rat. Arch Int Pharmacodyn Ther. 1986;283:312-320.

30. Savineau JP, Marthan R. Diosmin-induced increase in sensitivity to $\mathrm{Ca} 2+$ of the smooth muscle contractile apparatus in the rat isolated femoral vein. $B r J$ Pharmacol. 1994;111:978-980. doi:10.1111/ j.1476-5381.1994.tb14838.x

31. Bodinier M, Ly S, Finet M, Jean T. Etude in vitro de l'effet antiinflammatoire de la diosmine d'hémisynthèse ${ }^{\circ}$ dans un système de co-culture de cellules endothéliales veineuses et de granulocytes humains. [In vitro study of the anti-inflammatory effect of hemisynthetic diosmin in a co-culture system of human venous endothelial cells and granulocytes]. Artères et Veines. 1994;XIII:124-128. 
32. Bogachev VY, Boldin BV, Lobanov VN. Benefits of micronized purified flavonoid fraction as adjuvant therapy on inflammatory response after sclerotherapy. Int Angiol. 2018;37:71-78. doi:10.23736/S0392-9590.17.03868-8

33. Rabe E, Agus GB, Roztocil K. Analysis of the effects of micronized purified flavonoid fraction versus placebo on symptoms and quality of life in patients suffering from chronic venous disease: from a prospective randomized trial. Int Angiol. 2015;34:428-436.

34. Danielsson G, Jungbeck C, Peterson K, Norgren L. A randomised controlled trial of micronised purified flavonoid fraction vs placebo in patients with chronic venous disease. Eur J Vasc Endovasc Surg. 2002;23:73-76. doi:10.1053/ejvs.2001.1531

35. Carpentier PH, Mathieu M. Evaluation de l'efficacité clinique d'un médicament veinotrope: les enseignements d'un essai thérapetique avec la diosmine d'hémisynthèse dans le syndrome des jambes lourdes. [Assessment of the clinical effectiveness of a venotropic drug: findings from a therapeutic trial with hemisynthetic diosmin in heavy legs syndrome]. J des Maladies Vasculaires (Paris). 1998;23:106-112.
36. Cazaubon M, Bailly C, Ducros E, Lancrenon S. Étude comparative d'acceptabilité, d'efficacité et de tolérance de deux formes galéniques de diosmine $600 \mathrm{mg}$, dans le traitement des symptômes de la maladie veineuse chronique. [Acceptability, efficacy and safety of two pharmaceutical forms of diosmin $600 \mathrm{mg}$ in patients with chronic venous disease: a comparative study]. Angéiologie. 2011;63:69.
Vascular Health and Risk Management

\section{Publish your work in this journal}

Vascular Health and Risk Management is an international, peerreviewed journal of therapeutics and risk management, focusing on concise rapid reporting of clinical studies on the processes involved in the maintenance of vascular health; the monitoring, prevention and treatment of vascular disease and its sequelae; and the involvement
Dovepress

of metabolic disorders, particularly diabetes. This journal is indexed on PubMed Central and MedLine. The manuscript management system is completely online and includes a very quick and fair peerreview system, which is all easy to use. Visit http://www.dovepress. com/testimonials.php to read real quotes from published authors. 